RAD Conference Proceedings, vol. 2, pp. 25-29, 2017

www.rad-proceedings.org

\title{
THE FOCAL BRAIN PROTON BEAM IRRADIATION INSULT IN RATS - INDUCED MEMORY DISTURBANCE RELATED CHANGE IN ACETYLCHOLINE RECEPTOR BINDING
}

\author{
Nobuhiko Takai ${ }^{*}$, Masatsugu Ohgami' ${ }^{1}$ Koichi Ando², Akiko Uzawa3, \\ Ryoichi Hirayama 3 , Saori Nakamura', Yoshihito Ohba'
}

\author{
${ }^{1}$ Department of Analytical Chemistry, Faculty of Pharmaceutical Sciences, Nagasaki International University, \\ Nagasaki, Japan \\ ${ }^{2}$ Heavy Ion Medical Center, Gunma University, Japan \\ 3Medical Physics Research Program, Research Center for Charged Particle Therapy, National Institute of Radiological \\ Sciences (NIRS), Chiba, Japan
}

\begin{abstract}
Cerebral dysfunction is one of the major concerns associated with radiotherapy of brain tumours. However, little is known about the neurochemical basis of brain dysfunction induced by proton irradiation. We here investigated the early consequences of brain damages caused by a proton beam. Brains of male wistar rats were locally irradiated with a $70 \mathrm{MeV}$ proton beam. The irradiation dose was set at level known to produce vascular change followed by necrosis, which appeared the late period after irradiation with $30 \mathrm{~Gy}$. The eight-arm radial maze task in irradiated rats was used. In order to assess the preservation (recall) of memory, the rats that showed the spatial cognition were irradiated. The impairment of the preservation memory was not observed in the irradiated rats compared to the control ones 24 hrs after irradiation. Repeated measures of two-way ANOVA of correct choices and number of errors showed no differences between the control group and 30 Gy irradiated group. In order to assess the acquisition process of memory and working memory for the platform location in the water maze, the task was started on the 24 hrs after irradiation. In the learning task (the acquisition process of memory), there was no difference between the control group and irradiated group in the latency to platform. The rats that memorized the location of the standard position were irradiated, and the impairment of the long-term memory was not observed in the irradiated rats compared to the control ones $24 \mathrm{hrs}$ after irradiation. However, the irradiated rats required a substantially longer time finding out the platform than the control rats when the platform was placed in a non-standard position. From this it follows that a proton dose of 30 Gy impaired the working memory of rats. The function of muscarinic acetylcholine receptors was analyzed by in vivo binding assay using radioligand quinuclidinyl benzilate ([3H]QNB). The irradiated rats were intravenously injected with $5.5 \mathrm{MBq}$ of $[3 \mathrm{H}] \mathrm{QNB}$ on the 24 hrs after the irradiation. Autoradiographic studies showed a transitional increase of [3H]QNB in-vivo binding in the early phase after proton irradiation. On the other hand, no change in in-vitro [3H]QNB binding was seen in the autoradiogram of brain slices from the irradiated rats. The cerebral blood flow and the histopathological change in the brain appeared at 5 or 6 months after irradiation. These results indicate that the relation between behavioral impairment caused by radiation is closely related to the early change in the receptor function which could be detected in in-vivo conditions.
\end{abstract}

Key words: Radiotherapy, brain, memory disturbance, muscarinic acetylcholine receptors

DOI: $10.21175 /$ RadProc.2017.06

\section{INTRODUCTION}

Cerebral dysfunction is one of the major concerns associated with the radiotherapy of brain tumors and acute lymphoblastic leukemia. Side effects, such as learning impairment and cognitive dysfunction, are reported on the central nervous system (CNS) tissue after the exposure to radiation during cancer therapy (1-4). Children with acute lymphoblastic leukaemia who receive whole-brain irradiation often develop a neuropsychological deficit and learning disability $(5,6)$. The cognitive impairment stated above may relate to the hippocampus damage caused by X-rays, because the hippocampus is critical for the spatial navigation and the cognition functions in animals and humans (7-9).

Radiation damage of the CNS in animal models are reported for low linear energy transfer (LET) beams irradiation, such as $\mathrm{X}$ and gamma rays. However, there are few reports of a radiation injury after high-LET which, in general, produces stronger biological effectiveness than low-LET beams and is used to treat brain tumour. Therefore, it is important to determine the effect of the proton beam on the brain function.

The radiation-induced CNS damage generally appears from several months to years after irradiation. Late radiation-damage is mainly restricted to white matter and vasculature effects including edema,

*n takai@niu.ac.jp 
necrosis and demyelination (10-12). Late damages are a limiting factor in radiotherapy. The extent and latent period between the exposure and appearance of brain-damage legions appear to be dependent upon the radiation dose in animal models. The pathogenesis of the radiation injury is categorized into the following two theories $(12,13)$ : (1) that demyelination of axons and white matter necrosis gradually develop with time; and (2) that irradiation primarily damages the vasculature that, in turn, induces ischemia following by necrosis. Histopathological and behavioral changes are main end points to investigate the mechanisms of the delayed-irradiation brain damage in animal models.

Many reports demonstrate that the progressive deterioration of memory function over 7 months after whole-brain irradiation with 20-30 Gy of X-rays in rats $(13,14)$, using the water maze task. However, there are few studies on the learning ability and early effect by proton beam irradiation, a high LET beam has been reported to have greater biological effects on CNS than X-ray. Therefore, the aim of the present study is to assess early effects of local irradiation with proton beams on the learning ability with $\mathrm{ACh}$ receptor binding.

\section{MATERIALS AND METHODS \\ 2.1. Animals and irradiation procedure}

Male Wistar rats (8-9 weeks of age) were anesthetized with $50 \mathrm{mg} / \mathrm{kg}$ of pentobarbital. A group of rats was fixed horizontally on the plastic board and exposed to $70 \mathrm{MeV}$ energy proton beams. The deterioration of the eyesight and the decline of the muscle force influence the memory assessment, the water maze task can not be examined when irradiation disordered a motor function and life activity. Therefore, the whole brain, excluding the eyes and brain stem, was irradiated through a circular collimator $(10 \mathrm{~mm}$ diameter) in Fig. 1.



Figure 1. The irradiated area
The irradiated area was adjusted to be at the depth of $0-6 \mathrm{~mm}$ from the surface of skin (Fig. 1B). The control rats were also anaesthetized and received sham irradiation. Details of the irradiation procedures are given in Takahashi et al (15). All animal experiments were carried out with the permission and under the regulation of the Institutional Committee for Animal Safety and Welfare of the National Institute of Radiological Sciences, and in accordance with the Regulations on Appropriate Animal Breeding and Treatment, Ministry Office of Japan.

\subsection{Eight-arm radial maze and water maze task}

The eight-arm radial maze task was used in irradiated rats. In order to assess the long-term memory, the rats that memorized the spatial cognition were irradiated with proton beam, it were examined from 24 hours later (Fig. 2).

\section{Preservation (Recall) of memory 24hr after irradiation}
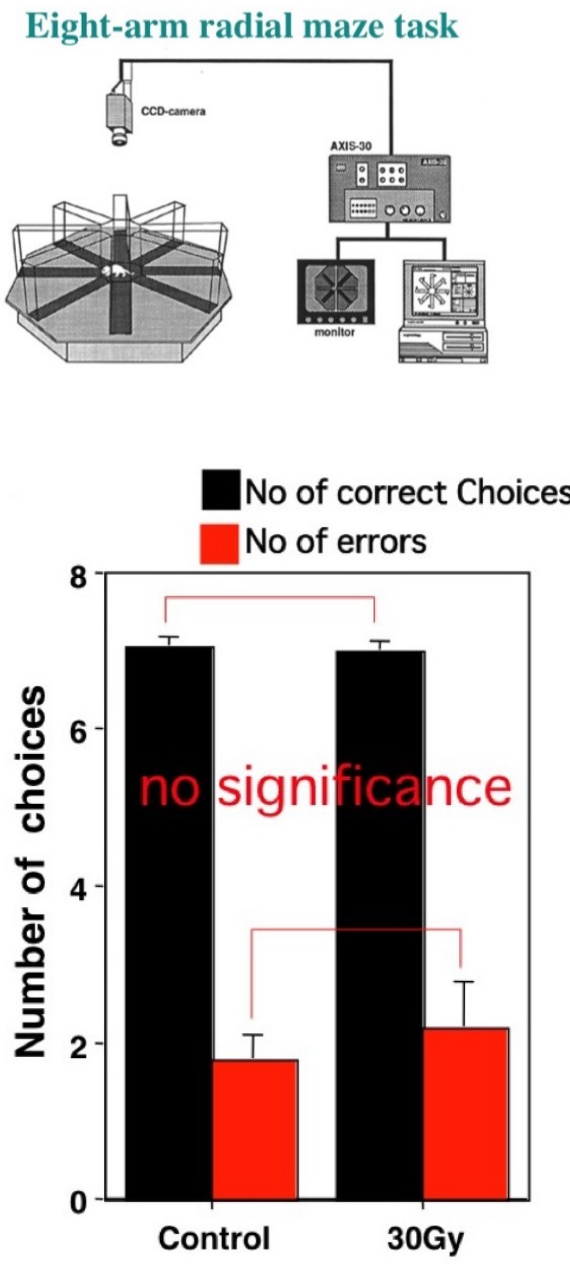

Figure 2. Eight-arm radial maze task

Irradiated and control rats were trained to find a clear Plexiglas platform (9 $\mathrm{cm}$ diameter) submerged 1.5 $\mathrm{cm}$ under the surface of water in a Morris water maze 
(120 $\mathrm{cm}$ diameter, $50 \mathrm{~cm}$ high, with $25 \mathrm{~cm}$ depth of water). The water maze was filled with room temperature water. The swim path was recorded by a CAT-10 image analyzing system (MUROMACHI CO., LTD, JAPAN), which computed the following 3 parameters, the latency to reach the platform, distance swum, and swim speed. Swim speed (distance/latency) provided an index of motor function. The latency to reach the platform was recorded with the cut-off time platform within $120 \mathrm{~s}$, it was placed on the platform and forced to remain there for $30 \mathrm{~s}$.

The reference and working memory task were used to assess the preservation (recall) of memory at the early phase after irradiation. The rats that well-memorized the location of the standard position and could reach the platform within 20 seconds were irradiated with proton ions. The reference and working memory were assessed at $24 \mathrm{hr}$ after irradiation. For the reference memory task, the platform was placed in standard positions and rats were given four trials. For the working memory task, the platform was placed in non-standard positions and rats were given four trials. The first trial in the working memory task provided information about the platform location and rats were required to match this position on the subsequent trials. The working memory was assessed by the improvement in the latency between Trial 1 and the matching to position in Trial 2.

\section{A Learning task}


Figure 3. Water maze task

\subsection{ACh receptor binding assay}

The animals were fixed horizontally, and focal irradiation was performed on the right-brain hemisphere (circular field, $10 \mathrm{~mm}$ in diameter) with a $70-\mathrm{MeV}$ proton beam. For the in-vitro binding study, brain slices were prepared from irradiated rats $(24 \mathrm{hr}$ post-radiation), and incubated with $1 \mathrm{nM}$ of $[3 \mathrm{H}] \mathrm{QNB}$ for $60 \mathrm{~min}$ at $4^{\circ} \mathrm{C}$, and autoradiograms were obtained. The in-vivo binding of $\left[{ }^{3} \mathrm{H}\right]$ QNB was measured by autoradiography. In the autoradiography study, irradiated rats were intravenously injected with 5.5 $\mathrm{MBq}$ of $[3 \mathrm{H}] \mathrm{QNB}$ at $24 \mathrm{hr}$ or 2 weeks after the irradiation, and decapitated 60 min after the tracer injection. The brains were quickly removed and frozen. Coronal sections (20 micro-m) were prepared and placed in contact with an imaging plate. The regions of interest were drawn on the autoradiograms, and the quantitative analysis of radioactivity in each region was performed.

\section{Irradiation side}



Figure 4. ACh receptor binding assay in vivo

\subsection{Statistical analysis}

Results were expressed as means \pm SEM. The significance of differences between the control group and irradiated group was analyzed with the unpaired t-test for the preservation of memory, reference memory and working memory task. Two-way repeated ANOVA was used for each parameter of the acquisition of place task. A P-value of less than 0.05 was considered to be statically significant.

\section{RESULTS AND DISCUSSION}

\subsection{Eight-arm radial maze and water maze task}

The eight-arm radial maze task in irradiated rats was used. In order to assess the long-term memory, the rats that memorized the spatial cognition were irradiated. At $24 \mathrm{hr}$ after irradiation, the impairment of the long-term memory was not observed in the irradiated rats compared to the control. Repeated measures of two-way ANOVA of correct choices and number of errors showed no differences between the control group and 30 Gy irradiated group (Fig. 2). 
In the water maze task, animals were assessed $24 \mathrm{hr}$ after irradiation, to assess the early-term effects of radiation-induced cerebral dysfunction. The acquisition of the place task from $24 \mathrm{hr}$ after irradiation, latency to reach the platform was not different between the control $(n=8)$ and irradiated group $(n=12)$. In the working memory task, control rats showed matching the position and rapid spatial learning, so that latency to the platform decreased significantly on trial 2 . Post-hoc analysis revealed that the swimming time during the first and second trials (first: $\mathrm{P}=0.374$; second: $\mathrm{P}<0.05$ for unpaired t-test) was longer in the irradiated group than the control group. These results indicated that the irradiated group was selectively impaired in the working memory, but not the reference memory. Many reports suggest that the radiation-induced damage generally develops from several months to years after irradiation $(12-14,16)$. Behavioral and histological studies of the early effect are rarely reported. Behavioral testing in the water maze at 24 hrs after irradiation, learning ability showed no difference between the control group and irradiated group. However, we found that the irradiated rats showed the definitive impairment of the working memory $24 \mathrm{hrs}$ after irradiation in the water maze, did not recall the spatial information from the long-term memory (reference memory). However, the neuronal degeneration was not observed in the hippocampus.

\subsection{ACh receptor binding assay}

We reported that no significant differences of in vitro binding of $\left[{ }^{3} \mathrm{H}\right] \mathrm{QNB}$ were seen between the irradiated and non-irradiated sides (17). As shown in Fig. 4, a significant increase in radioactivity in the cerebral cortex was observed in the irradiated side of the intact brain $24 \mathrm{hrs}$ after irradiation. This accumulation seemed to be due to a increase in the specific binding of $\left[{ }^{3} \mathrm{H}\right] \mathrm{QNB}$ to muscarinic acetylcholine receptors. An increase in [3H]QNB binding induced by proton-beam irradiation was transiently observed in the early phase after irradiation. The most important finding is that an increase in $\left[{ }^{3} \mathrm{H}\right]$ QNB binding caused by irradiation can only be detected in the intact brain. In the cholinergic system, such as choline acethyltransferase and ACh receptor, it plays important roles in learning and spatial cognition (18-20). We indicated that a transitional increase of $\mathrm{mACh}$ receptor in vivo binding in the early phase at 24 hrs after irradiated with the proton beam at a dose of 30 Gy. No change in in-vitro mACh receptor binding is seen in autoradiogram of brain slices from irradiated rats. The cerebral blood flow and the histlogical features of the brain were also changed at 5 or 6 months post-irradiation. These data suggest that the relation between behavioral impairment caused by radiation is closely related to an early change in the receptor function which could be detected in in-vivo condition. These results furthermore indicated that the deficit in the working memory may be detected in the absence of histopathological damage, associated with the neurogenesis and neuronal receptor function.

\section{CONCLUSIONS}

We demonstrated that irradiated rats showed the definitive impairment of the working memory $24 \mathrm{hr}$ after irradiation in the water maze, while neuronal degeneration was not observed. However, the early changes in ACh receptor function induced by irradiation were only detected in vivo. These results indicated that the relation between the behavioral impairment caused by radiation is closely related to the early change in receptor function which could be detected in in-vivo condition. The neuro-receptor imaging with positron emission tomography (PET) or single photon emission tomography (SPECT) enabled the evaluation of receptor function in the living human brain. The aim of this study was to develop a method for the early detection of brain dysfunction caused by irradiation, by examining whether the receptor binding in the intact brain is altered by irradiation.

Acknowledgement: This work was performed as part of a research project involving the Heavy-Ion Medical Accelerator in Chiba (HIMAC, National Institute of Radiologic Sciences).

\section{REFERENCES}

1. M. Schmidinger et al., "Psychometric- and quality-of-life assessment in long-term glioblastoma survivors." J. Neurooncol., vol. 63, no. 1, pp. 55-61, May 2002.

DOI: $10.1023 / \mathrm{A}: 1023740303162$

2. J. P. Imperato et al., "Effects of treatment on long-term survivors with malignant astrocytomas," Ann. Neurol., vol. 28, no. 6, pp. 818-822, Dec. 1990.

DOI: $10.1002 /$ ana.410280614 PMid: 2178330

3. P. Salander et al., "Long-term memory deficits in patients with malignant gliomas," J. Neuro-Oncology., vol. 25 , no. 3, pp. 227-238, Oct. 1995

DOI: $10.1007 / \mathrm{BFO} 1053156$ PMid: 8592173

4. R. J. Packer et al., "A prospective study of cognitive function in children receiving whole-brain radiotherapy and chemotherapy: 2-year results," J. Neurosurg., vol. 70, no. 5, pp. 707-713, May 1989.

DOI: $10.3171 /$ jns.1989.70.5.0707

PMid: 2709111

5. C. Eiser et al., "Learning difficulties in children treated for acute lymphoblastic leukaemia (ALL)," Pediatr. Rehabil., vol. 4, no. 3, pp. 105-118, Jul-Sep. 2001. DOI: $10.1080 / 13638490110064806$ PMid: 11831563

6. A. Davidson et al., "Functional neurological outcome in leukaemic children receiving repeated cranial irradiation," Radiother Oncol., vol. 31, no. 2, pp. 101-109, May 1994. DOI: 10.1016/0167-8140(94)90389-1

7. M. S. Zola et al., "Impaired Recognition Memory in Monkeys after Damage Limited to the Hippocampal Region," J. Neurosci., vol. 20, no. 1, pp. 451-463, Jan. 2000.

Retrieved from:

http://www.jneurosci.org/content/20/1/451.long

8. R. Liscak et al., "Leksell gamma knife lesioning of the rat hippocampus: the relationship between radiation dose and functional and structural damage," J. Neurosurg., vol. 97, no. 5, pp. 666-673, Dec. 2002. DOI: $10.3171 /$ jns.2002.97.supplement PMid: 12507117

9. M. C. Cheung et al., "Memory impairment in humans after bilateral damage to lateral temporal neocortex," Neuroreport., vol. 14, no. 3, pp. 371-374, Mar. 2003. DOI: 10.1097/01.wnr.0000057865.05120.f3 PMid: 12634486 
10. H.S. Reinhold et al., "Development of blood vessel-related radiation damage in the fimbria of the central nervous system," Int J Radiat Oncol Biol Phys., vol. 18, no. 1, pp. 37-42, Jan. 1990.

DOI: $10.1016 / 0360-3016(90) 90264-\mathrm{K}$

11. G. J. M. J. van den Aardweg et al., "A new model of radiation-induced myelopathy: A comparison of the response of mature and immature pigs," Int. J. Radiat. Oncol. Biol. Phys., vol. 29, no. 4, pp. 763-770, Dec. 1994. DOI: 10.1016/0360-3016(94)90564-9

12. W. Calvo et al., "Time- and dose-related changes in the white matter of the rat brain after single doses of X rays," Br. J. Radiol., vol. 61, no. 731, pp. 1043-1052, Nov. 1988.

DOI: $10.1259 / 0007-1285-61-731-1043$ PMid: 3208008

13. H. Hodges et al., "Late behavioural and neuropathological effects of local brain irradiation in the rat," Behav. Brain Res., vol. 91, no. 1-2, pp. 99-114, Dec. 1998. DOI: $10.1016 /$ So166-4328(97)00108-3

14. I. Lamproglou et al., "Radiation-induced cognitive dysfunction: an experimental model in the old rat," Int. J. Radiat. Oncol. Biol. Phys., vol. 31, no. 1, pp. 65-70, Jan. 1995. DOI: $10.1016 / 0360-3016(94) 00332-F$

15. S. Takahashi et al., "Histological and elemental changes in the rat brain after local irradiation with carbon ion beams," J. Radiat. Res. vol., 43, no. 2, pp. 143-152, Jun. 2002.

DOI: $10.1269 /$ jrr. 43.143
16. Y. Yoneoka et al., "An experimental study of radiation-induced cognitive dysfunction in an adult rat model," Br. J. Radiol., vol. 72, no. 864, pp. 1196-1201, Dec. 1999.

DOI: $10.1259 /$ bjr.72.864.10703477

PMid: 10703477

17. O. Inoue et al., "An increase in $[3 \mathrm{H}] \mathrm{QNB}$ binding by proton-beam irradiation in intact rat brain: an apparent positive cooperativity of binding," Neurosci. Lett., vol. 250, no. 1, pp. 33-36, Jun. 1998. DOI: $10.1016 /$ So304-3940(98)00426-1

18. S. Okada et al., "Histopathological and morphometric study of the late effects of heavy-ion irradiation on the spinal cord of the rat," Radiat. Res., vol. 150, no. 3, pp. 304-315, Dec. 1998.

Retrieved from: http://www.jstor.org/stable/3579980 Retrieved on: Jan. 19, 2017

19. S. Mizumatsu et al., "Extreme sensitivity of adult neurogenesis to low doses of X-irradiation," Cancer. Res., vol. 63, no. 14, pp. 4021-4027, Jul. 2003.

Retrieved from:

http://cancerres.aacrjournals.org/content/63/14/4021. long Retrieved on: Feb. 18, 2017

20. K. Mishima et al., "The scopolamine-induced impairment of spatial cognition parallels the acetylcholine release in the ventral hippocampus in rats," Jpn. J. Pharmacol., vol. 84, no. 2, pp. 163-173, Oct. 2000.

DOI: $10.1254 /$ jjp.84.163

PMid: 11128039 